\title{
Relativistic and Newtonian Shock Breakouts
}

\author{
Ehud Nakar \\ Raymond and Beverly Sackler School of Physics \& Astronomy, Tel Aviv University, \\ Tel Aviv 69978, Israel
}

\begin{abstract}
Observations of the first light from a stellar explosion can open a window to a wealth of information on the progenitor system and the explosion itself. Here I briefly discuss the theoretical expectation of that emission, comparing Newtonian and relativistic breakouts. The former takes place in regular core-collapse supernovae ( $\mathrm{SNe}$ ) while the latter is expected in $\mathrm{SNe}$ that are associated with gamma-ray bursts (GRBs), extremely energetic SNe (e.g., SN2007bi) and white dwarf explosions (e.g., type Ia and .Ia SNe, accretion induced collapse). I present the characteristic observable signatures of both types of breakouts, when spherical. Finally, I discuss Newtonian shock breakouts through wind, which produce a very luminous signal, with an X-ray component that is weak around the breakout, and becomes brighter afterwards.
\end{abstract}

Any observable stellar explosion must break out of the stellar surface. The breakout emission is the first light seen from an explosion, and it probes the outer layers of the star. The signal from a SN breakout was first calculated four decades ago. It was immediately realized that detection of the breakout emission, or of the emission that comes shortly after it, are excellent probes of progenitor properties such as its radius and mass, and the wind it throws just prior to its explosion. It is also a probe of the explosion energy and geometry. These are all important properties, which may, for example, shed light on the evolution of massive stars. It is therefore important to provide theoretical predictions of the observed signature of shock breakouts in different scenarios. Here I briefly summarize the main properties of shock breakout pulses in the cases of Newtonian and relativistic breakouts from the surface of a star and of a Newtonian breakout from a thick stellar wind. I consider only spherically symmetric cases. All the results described here, including references and comparison to previous studies, are discussed in length in Nakar \& Sari (2010), Nakar \& Sari (2012) and Svirski et al. (2012).

\section{Newtonian Breakout}

When a shock is generated within a stellar envelope it accelerates in the decreasing density as it approaches the stellar surface. The shock breaks out once $\tau=c / v_{s}$ where $\tau$ is the optical depth towards the observer, $c$ is the speed of light and $v_{s}$ is the shock speed. The total energy in the breakout pulse is therefore determined by the stellar radius $R_{*}$ and by the breakout velocity $v_{b o}$. It depends strongly on $R_{*}$, reaching $\sim 10^{48} \mathrm{erg}$ in a typical red-supergiant (RSG) SNe compared to $\sim 10^{45}$ erg in a typical Wolf-Rayet (WR) SNe. The duration of the breakout pulse is dominated by photon travel time and it is therefore (in spherical symmetry) simply $R_{*} / c$. The breakout luminosity, therefore, varies weakly between different progenitor radii and it is typically $\sim 10^{44}-10^{45} \mathrm{erg} / \mathrm{s}$. The breakout temperature depends strongly on $v_{b o}$. Slow breakouts, $v_{b o}<15,000 \mathrm{~km} / \mathrm{s}$ results in a UV flash with temperature $T_{b o}=10-100 \mathrm{eV}$, but faster shocks results in a $\mathrm{keV}$ or even harder breakouts. The reason is that the radiation behind fast shocks falls out of thermal equilibrium due to photon starvation. Thus, the small number of post shock photons shares the entire post shock energy, achieving a higher energy per photon. 
Relativistic Breakout

A key process in relativistic shock breakouts is the formation of pairs, which play two roles. First, pair formation regulates photon production in the shock, preventing the post shock temperature to climb above $\sim 200 \mathrm{keV}$. As a result and in contrast to Newtonian shocks, there is no strong dependence of the temperature immediately behind the shock on its velocity or Lorentz factor. Second, pairs significantly increase the optical depth of the plasma. As a result, the temperature at which the emission is released to the observer is set by pair annihilation to be $\sim 50 \mathrm{keV}$ in the outflow rest frame.

The physical setup after the shock end crossing the star is similar in some aspects to that of a pair-radiation, baryon loaded, fireball (as often discussed in the context of gamma-ray bursts). The pair loaded plasma accelerates as long as it is still opaque without significant loses of internal energy to bulk motion energy. In fact, as a result of the post shock energy density profile, the fastest moving parts at the front of the flow gain energy at the expense of slower moving parts that push from behind.

As discussed above the photons are released from the breakout layer once its rest frame temperature is $\sim 50 \mathrm{keV}$. Therefore the observed breakout temperature is $T_{b o} \approx 50 \gamma_{b o}$ $\mathrm{keV}$, where $\gamma_{b o}$ is the Lorentz factor of the breakout layer when it becomes transparent. the duration of the breakout pulse is set by photon travel time, $t_{b o} \approx R_{b o} / c \gamma_{b o}^{2}$. Calculating the energy in the breakout pulse, $E_{b o}$ is slightly more complicated, but it also depends only on $R_{b o}$ and $\gamma_{b o}$ (see Nakar \& Sari 2012). Therefore, there are three generic observables $\left(E_{b o}, t_{b o}\right.$ and $\left.T_{b o}\right)$ that depend on only two parameters $R_{b o}$ and $\gamma_{b o}$. Thus, the observed energy, temperature and time scales of the breakout overconstrain $R_{b o}$ and $\gamma_{b o}$, where any two observables out of these three are enough to determine both $R_{b o}$ and $\gamma_{b o}$. In case all three observables are measured, they must satisfy

$$
t_{b o} \sim 20 \mathrm{~s}\left(\frac{E_{b o}}{10^{46} \mathrm{erg}}\right)^{1 / 2}\left(\frac{T_{b o}}{50 \mathrm{keV}}\right)^{-\frac{9+\sqrt{3}}{4}},
$$

if the source of the flare is a quasi-spherical relativistic shock breakout. This relation can be used to test whether any observed gamma-ray flare is consistent with a relativistic breakout origin or not.

\section{Newtonian Breakout through a thick wind}

When an exploding star is surrounded by a wind that is thick enough, the radiation dominated shock remains confined to the wind after it crosses the star and the breakout takes place at $R_{b o} \gg R_{*}$. Here I shortly describe cases where the wind is so thick that the breakout takes place only days after the explosion. The breakout luminosity here is almost similar to that of the Newtonian breakout through a stellar surface, i.e., $\sim 10^{44}$ $\mathrm{erg} / \mathrm{s}$. But, given the long duration of the breakout pulse, the released energy is significantly larger. In the most extreme cases, when the breakout time is $\sim 60$ days, the entire explosion energy can be released in the breakout pulse. Thus, shock breakouts through a thick wind are promising candidates for some of the observed super-luminous SNe.

An interesting property of wind breakouts is that the shock makes a transition from radiation mediated to collisionless following the breakout. As a result the emission contains two spectral components - soft (optical/UV) and hard (X-rays and possibly soft gammarays). During the breakout, the soft component temperature can vary significantly from one event to another $\left(10^{4}-10^{6} \mathrm{~K}\right)$, where events with longer breakout time are generally softer. The hard component is always a small fraction, $\sim 10^{-4}$, of the breakout emission, and its fraction of the total luminosity rises quickly afterwards, becoming dominant at $\sim 10-50 t_{b o}$. Therefore, in addition for being extremely luminous, wind shock breakouts 
are expected to show very weak X-ray emission around the time that the optical-UV light curve peaks, but it should increase with time afterwards.

\section{Acknowledgement}

This research was partially supported by an ERC starting grant (GRB-SN 279369)

\section{References}

Nakar, E. \& Sari, R. 2010, ApJ, 725, 904

Nakar, E. \& Sari, R. 2012, ApJ, 747, 88

Svirski, G., Nakar, E., \& Sari, R. 2012, arXiv:1202.3437 